\title{
VARIABILIDADE ESPACIAL E TEMPORAL DO POTENCIAL MÁTRICO DA ÁGUA EM TERRA ROXA ESTRUTURADA
}

\author{
F.B.P. PACHECO; K. REICHARDT; R.L. TUON \\ Departamento de Flsica e Meteorologia, ESALQ/USP, C.P. 9, CEP: 13418-900 - Piracicaba,SP \\ O.O.S. BACCHI \\ Centro de Energia Nuclear na Agricultura, USP, C.P. 93, CEP: 13400-970 - Piracicaba,SP \\ M.M. VILLAGRA \\ University of Gent, Coupure Links 653, B-9000, Gent - Belgica
}

\begin{abstract}
RESUMO: As variabilidades de medidas de potencial mátrico da água no solo e de gradiente de potencial hidráulico são analisadas utilizando conceitos geoestatísticos e estatística da distribuição normal. São analisados 25 pares de dados, coletados ao longo de uma transecçāo de terra roxa estruturada, de $125 \mathrm{~m}$ de comprimento. Autocorrelogramas e semivariogramas não indicaram dependência espacial nas variáveis estudadas. A variabilidade do potencial mátrico da água no solo, medida através de tensiómetros, é menor do que a variabilidade do gradiente de potencial hidráulico. Este último apresentou alta variabilidade, a ponto de comprometer seu uso em estimativas de fluxos de água no solo.

Descrilores: variabilidade espacial, variabilidade temporal, autocorrelação, semivariograma, potencial mátrico, gradiente de potencial hidráulico.
\end{abstract}

\section{SPATIAL AND TEMPORAL VARIABILITY OF SOIL WATER MATRIC POTENTIAL IN A DARK RED LATOSOL.}

\begin{abstract}
ARSTRACT: Variabilities of soil water matric potential and of hydranlic gradients are analysed using geostatistical concepts and normal distribution statistics. Twenty five data pairs, collected along a $125 \mathrm{~m}$ transect of a dark red latosol (terra roxa estruturada) are analysed using autocorrelograms and semivariograms. No spatial dependence was found for both variables. The variability of soil water matric potential, measured by tensiometry, is less than the variability of hydraulic gradients. This last variable presented a high variability, to the point of compromising its use in soil water flux estimatives.
\end{abstract}

Key words: spatial variability, temporal variability, autocorrelation, semivariogram, matric potential, hydraulic gradient.

\section{INTRODUÇÃo}

Sendo o potencial mátrico da água no solo uma medida de seu estado de energia em relação ao estado da água livre e pura, sua importância na descrição dos processos dinâmicos que ocorrem no solo, torna-se indiscutível. Nas últimas décadas tem-se observado com maior ênfase a grande variabilidade, principalmente espacial, de propriedades do solo, ao ponto de mostrar sérias dificuldades no uso de dados coletados em condições de campo. Variabilidade no arranjo poroso de solos leva a diferentes condições de retenção de água $e$, consequentemente à variações de potencial mátrico. VILLAGRA et al. (1988) apresentam resultados detalhados sobre esta variabilidade e concluem que ela é fator importan- te, não podendo ser desprezada tanto em experimentação científica como em seu uso para manejo de solos. Em seu trabalho as medidas foram feitas com tensiomêtros instalados na zona arável do solo. Seus dados são portanto específicos para a camada superficial, mais sujeita à ações antrópicas e do tempo. O presente estudo é complementar ao de VILLAGRA et al. . (1988), envolvendo medidas em camadas mais profundas do solo, mais estáveis do ponto de vista estrutural e medidas ao longo de um periodo mais extenso (2 anos).

REICHARDT et al. (1990) abordam aspectos da dificuldade do uso de dados de potencial mátrico em cálculos de balanços hídricos e REICHARDT et al. (1993a) discutem o problema da dificuldade de quantificar fluxos de água no solo, chegando à conclusão de que não 
possível utilizar o modelo condutividade-gradiente de potencial (equação de Darcy) para estimar fluxos de água em condições de campo. Torna-se imperativo, portanto, caracterizar melhor a variabilidade dos diversos parâmetros que governam o movimento de água no solo. Este trabalho tem por objetivo melhorar a caracterização das variabilidades temporal e espacial do potencial mátrico da água no solo, para viabilizar seu uso na solução de problemas ligados ao manejo agrícola de solos.

\section{MATERIAL E METTODOS}

O experimento foi conduzido no campo experimental da ESALQ/USP, município de Piracicaba, S.P., em terra roxa estruturada, classificada como Rhodic Kandiudalf. Trata-se de solo argiloso, com $64.0,7.5$ e $28.5 \%$ de argila, limo e areia, respectivamente. $O$ perfil é profundo, com lençol freático a vários metros de profundidade.

O potencial mátrico h $\left(\mathrm{cm} \mathrm{H}_{2} \mathrm{O}\right.$ ) foi medido com tensiomêtros de manômetro de mercúrio, instalados nas profundidades de $135 \mathrm{e}$ $165 \mathrm{~cm}$ a partir da superfície do solo. Vinte e cinco conjuntos de tensiômetros foram instalados de $5 \mathrm{em}$ 5 metros ao longo de uma transceção de $125 \mathrm{~m}$, em área praticamente horizontal do campo experimental. As medidas foram feitas por um período de mais de 2 anos (4/2/89 a 14/3/91) com freqüências diárias, semanais ou quinzenais, dependendo da situação do campo. Durante os dois anos houve períodos de pousio, solo arado e gradeado, cobertura morta, milho, etc. As medidas de potencial mátrico foram feitas nas profundidades indicadas com o objetivo de estudar gradientes de potencial hidráulico $\mathrm{\nabla H}$ na profundidade $\mathrm{z}=150$ $\mathrm{cm}$, por diferenças finitas, de acordo com:

$$
\nabla H=\frac{h_{135}-h_{165}}{30}+1
$$

Com este critério, valores positivos de $\mathrm{WH}$ indicam fluxo descendente $\mathrm{e}$ valores negativos ascendente.

As variabilidades espaciais e temporais do potencial mátrico $h$ e do gradiente de potencial total $\nabla \mathrm{H}$ foram estudadas empregando a estatística da distribuição normal e a geoestatística. Para isto foram empregados os programas LOTUS e STATGRAPHICS que permitem a análise de distribuições, obtenção de momentos da distribuição normal, testes de significância e semivariogramas.

\section{RESULTADOS E DISCUSSÃO}

Uma visão geral da variabilidade espacial dos dados de potencial matricial ao longo da transeção, na profundidade de $135 \mathrm{~cm}$, pode ser vista na Figura 1, para três situações nas quais o valor médio das $\mathbf{2 5}$ medidas representava condições de solo cada vez mais seco. Nesta profundidade relativamente grande, raramente foram encontrados valores de potencial mátrico inferiores a $-400 \mathrm{~cm} \mathrm{H}_{2} \mathrm{O}$.

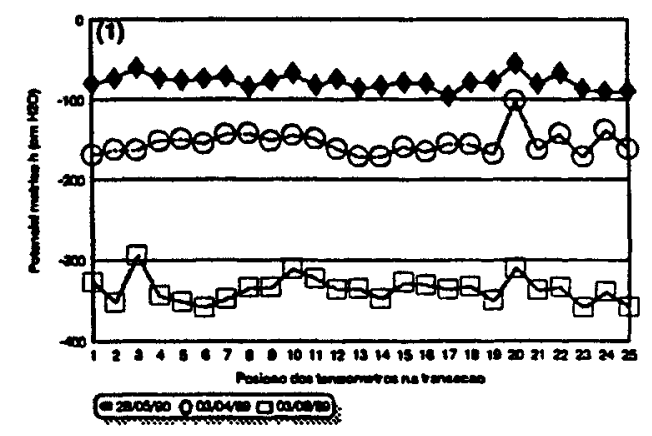

Figura 1 - Distribuição do potencial mátrico da água no solo, na profundidade de 135 $\mathrm{cm}$, em três datas selecionadas, representando situações de solo cada vez mais seco.

Para verificar a existência de uma dependência espacial nos dados de $h$ procedeu-se à elaboração de autocorrelogramas (Figura 2) e de semivariogramas (Figura 3). Vê-se claramente que não existe dependência espacial significativa já para os primeiros vizinhos. Os semivariogramas tendem imediatamente para a variância da população, como seria esperado em caso de não-dependência espacial.

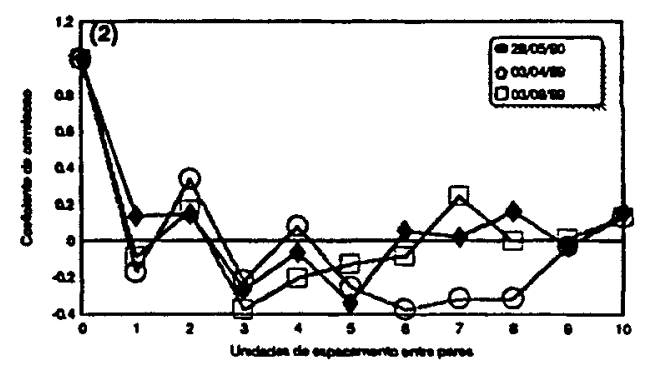

Figura 2 - Autocorrelogramas dos dados da Figura 1 , de potencial mátrico da água no solo. Uma umidade de espaçamento (lag) equivale a $5 \mathrm{~cm}$. 
TABELA 1 - Potencial mátrico do solo ao longo de uma transeção de 25 pontos, em três dias distintos, com suas respectivas estatísticas básicas e o número de amostras $\mathrm{N}$ de acordo com a equação 2 .

\begin{tabular}{|c|c|c|c|}
\hline Pontos & $28 / 05 / 90$ & $03 / 04 / 89$ & $03 / 08 / 89$ \\
\hline 1 & -79.4 & -168.9 & -326.4 \\
\hline 2 & -73.4 & -162.6 & -351.9 \\
\hline 3 & -60.5 & -163.9 & -293.6 \\
\hline 4 & -73.4 & -151.5 & -343.0 \\
\hline 5 & -75.9 & -149.0 & -350.6 \\
\hline 6 & -74.7 & -155.3 & -358.2 \\
\hline 7 & -71.4 & -143.2 & -347.3 \\
\hline 8 & -83.7 & -141.7 & -333.2 \\
\hline 9 & -75.9 & -151.5 & -333.0 \\
\hline 10 & -66.8 & -145.0 & -310.0 \\
\hline 11 & -82.2 & -149.0 & -321.6 \\
\hline 12 & -74.7 & -161.6 & -335.5 \\
\hline 13 & -86.0 & -171.7 & -334.2 \\
\hline 14 & -83.5 & -171.7 & -346.8 \\
\hline 15 & -78.4 & -159.1 & -326.7 \\
\hline 16 & -80.7 & -165.1 & -330.2 \\
\hline 17 & -96.1 & -155.3 & -335.5 \\
\hline 18 & -78.4 & -156.6 & -331.7 \\
\hline 19 & -77.2 & -167.9 & -349.3 \\
\hline 20 & -55.8 & -101.1 & -309.0 \\
\hline 21 & -81.0 & -162.9 & -336.7 \\
\hline 22 & -67.1 & -142.7 & -333.0 \\
\hline 23 & -87.0 & -171.4 & -357.9 \\
\hline 24 & -90.3 & -138.2 & -338.5 \\
\hline 25 & -89.5 & -161.3 & -356.6 \\
\hline média & -77.7 & -154.7 & -335.6 \\
\hline variância & 80.5 & 217.5 & 240.1 \\
\hline desvio & 9.0 & 14.7 & 15.5 \\
\hline c.v. & -11.5 & -9.5 & -4.6 \\
\hline máximo & -55.8 & -101.1 & -293.6 \\
\hline mínimo & -96.1 & -171.7 & -358.2 \\
\hline$N(10 \%)$ & 5 & 3 & 1 \\
\hline $\mathrm{N}(5 \%)$ & 20 & 14 & 3 \\
\hline
\end{tabular}


TABELA 2 - Gradiente de potencial hidráulico ao longo de uma transeção de 25 pontos, na profundidade de $150 \mathrm{~cm}$, em três dias distintos, com suas respectivas estatísticas básicas e o número de amostras $\mathbf{N}$ de acordo com a equação 2.

\begin{tabular}{|c|c|c|c|}
\hline Pontos & $28 / 05 / 90$ & $03 / 04 / 89$ & $03 / 08 / 89$ \\
\hline 1 & 1.218 & 1.504 & 1.134 \\
\hline 2 & 1.050 & 1.252 & 1.041 \\
\hline 3 & 0.723 & 2.890 & 1.059 \\
\hline 4 & 1.293 & 1.546 & 1.125 \\
\hline 5 & 1.260 & 1.210 & 0.924 \\
\hline 6 & 1.251 & 0.790 & 1.083 \\
\hline 7 & 1.671 & 1.336 & 1.461 \\
\hline 8 & 1.713 & 1.378 & 0.873 \\
\hline 9 & 1.713 & 1.514 & 1.083 \\
\hline 10 & 1.571 & 2.428 & 1.193 \\
\hline 11 & 1.470 & 1.588 & 1.176 \\
\hline 12 & 1.428 & 1.840 & 1.134 \\
\hline 13 & 1.377 & 1.798 & 1.587 \\
\hline 14 & 0.999 & 1.462 & 0.957 \\
\hline 15 & 1.302 & 2.050 & 1.176 \\
\hline 16 & 1.260 & 2.008 & 1.092 \\
\hline 17 & 1.419 & 2.218 & 0.873 \\
\hline 18 & 1.881 & 1.840 & 1.377 \\
\hline 19 & 1.134 & -1.336 & 0.798 \\
\hline 20 & 3.645 & 2.806 & 2.511 \\
\hline 21 & 1.554 & 1.714 & 1.008 \\
\hline 22 & 1.647 & 1.924 & 0.765 \\
\hline 23 & 1.227 & 1.630 & 1.185 \\
\hline 24 & 1.705 & 1.672 & 1.033 \\
\hline 25 & 1.335 & 1.252 & 1.125 \\
\hline média & 1.474 & 1.719 & 1.151 \\
\hline variância & 0.263 & 0.230 & 0.111 \\
\hline desvio & 0.513 & 0.480 & 0.333 \\
\hline c.v. & 34.812 & 27.903 & 28.967 \\
\hline máximo & 3.645 & 2.890 & 2.511 \\
\hline mínimo & 0.723 & 0.790 & 0.765 \\
\hline$N(10 \%)$ & 47 & 30 & 32 \\
\hline $\mathrm{N}(5 \%)$ & 186 & 120 & 129 \\
\hline
\end{tabular}




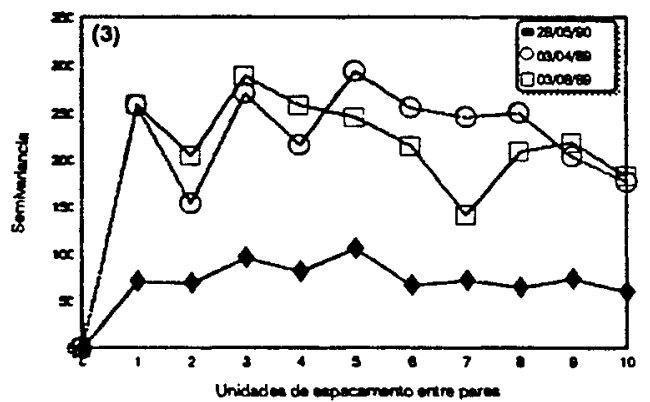

Figura 3: Semivariogramas dos dados da Figura 1, de potencial mátrico da água no solo. Uma unidade de espaçamento (lag) equivale a $5 \mathrm{~m}$.

A Figura 4 mostra histogramas de freqüência e gráficos de probabilidade normal para as 25 amostras da Figura 1. Para o solo mais úmido (h em torno de $-77 \mathrm{~cm} \mathrm{H}_{2} \mathrm{O}$, Figuras $4 \mathrm{a} \mathrm{e} 4 \mathrm{~b}$ ) nota-se que os dados parecem seguir a distribuição normal. Com o secamento do solo $(\mathrm{h}=-154 \mathrm{e}$ $-335 \mathrm{~cm} \mathrm{H}_{2} \mathrm{O}$ Figs $4 \mathrm{c}, 4 \mathrm{~d}, 4 \mathrm{e}$ e $4 \mathrm{f}$ ) torna-se nítido o desvio do comportamento normal, até que para $\mathrm{h}=-335 \mathrm{~cm} \mathrm{H} \mathrm{O}$ a distribuição foge completamente da normal. Apesar destas limitações foi feita análise de variância $\mathrm{e}$ os dados encontram-se na TABELA 1. O C.V. foi maior para o solo mais úmido $(11,5 \%)$ e menor para o solo mais seco $(4,6 \%)$. Usando a metodologia proposta por WARRICK \& NIELSEN (1980) pode-se estimar o número $\mathrm{N}$ de tensiômetros (medidas) necessários para obter um valor médio $h^{*}$ com uma precisão pré estabelecida. Segundo estes autores, o número $\mathrm{N}$ de amostras necessárias para a nova média estar dentro de d unidades da média, dentro de um nível de significância $x \alpha$ escolhido, é dado por:

$$
N=x_{\alpha}^{2} \frac{s^{2}}{d^{2}}
$$

sendo $\mathrm{x}_{\alpha}$ obtido da tabela $\mathrm{t}$ de Student para infinitos graus de liberdade, e s o desvio padrão.

Sci. agric., Piracicaba, 51(2):327-334, maio/ago., 1994

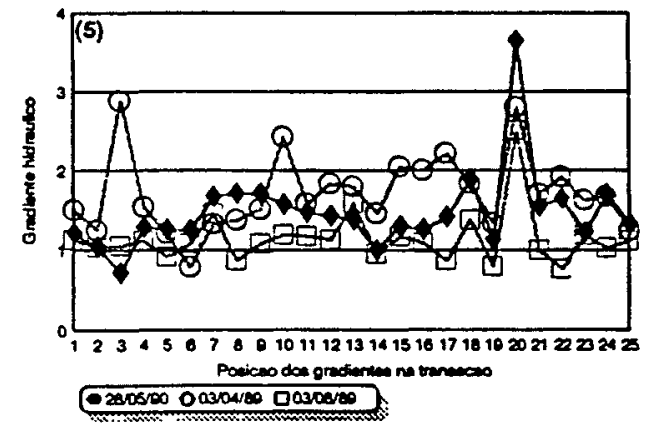

Figura 5: Distribuição do gradiente hidráulico, na profundidade de $150 \mathrm{~cm}$, calculado pela equação 1 , em três datas selecionadas, representando situações de solo cada vez mais seco.

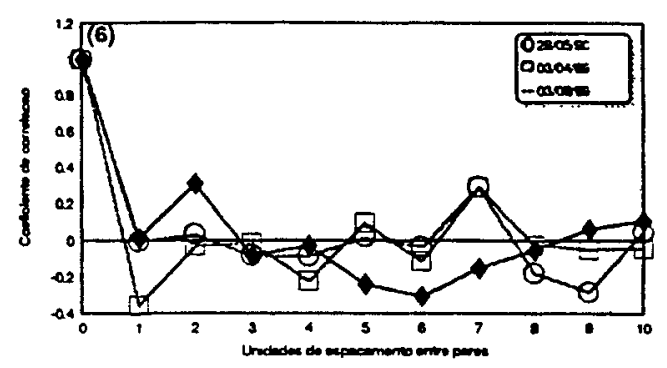

Figura 6: Autocorrelogramas dos dados da Figura 5, de gradiente hidráulico. Uma unidade de espaçamento (lag) equivale a $5 \mathrm{~m}$.

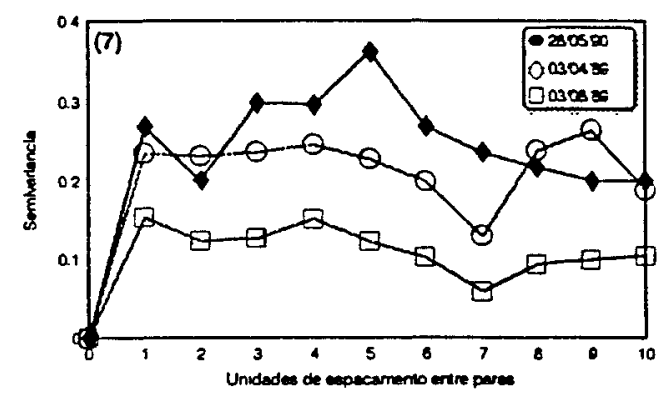

Figura 7: Semivariogramas dos dados da Figura 5, de gradiente hidráulico. Uma unidade de espaçamento (lag) equivale a $5 \mathrm{~m}$. 
FIGURA 4
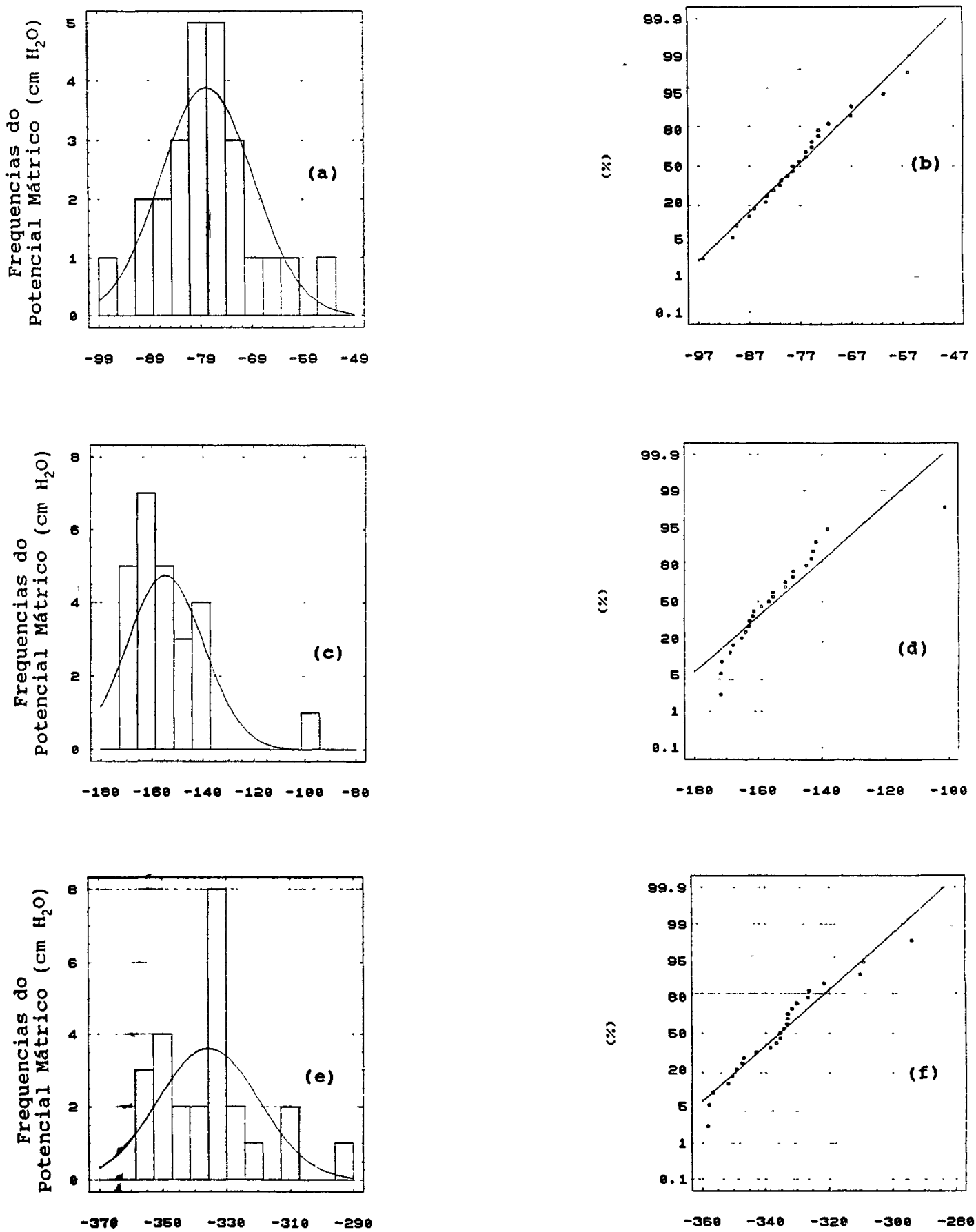

Figura 4: Histogramas de frequiência e gráficos de probabilidade normal dos dados de potencial mátrico, na profundidade de $135 \mathrm{~cm}$, em três datas selecionadas. 
FIGURA 8
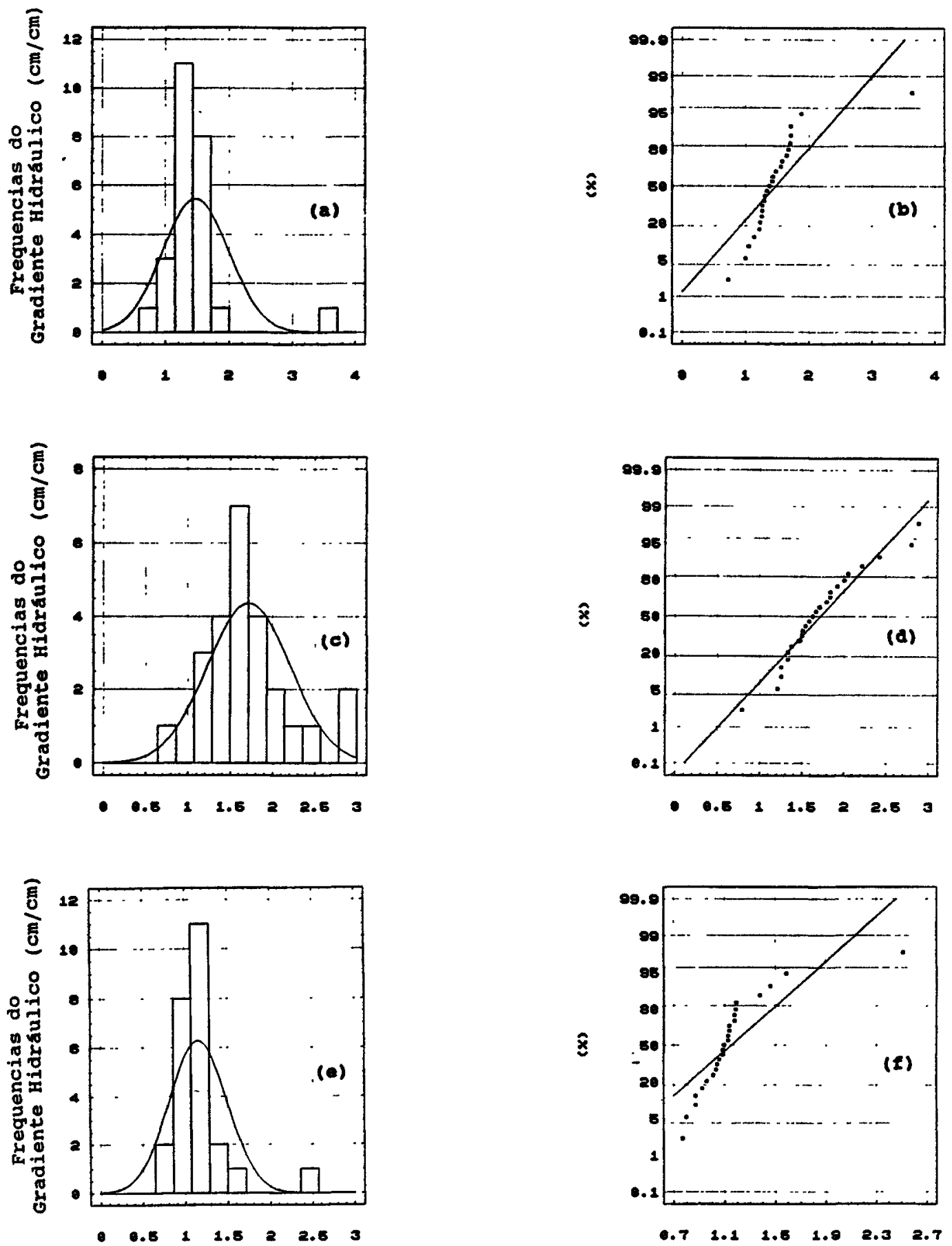

Figura 8: Histogramas de freqüência e gráficos de probabilidade normal dos dados de gradiente hidráulico, na profundidade de $150 \mathrm{~cm}$, em três datas selecionadas. 
Para os dados do Quadro 1, considerando os desvios d de $5 \%$, ou $0.05 \mathrm{~h}^{*}$ e um nível de probabilidade de $95 \%\left(x_{\alpha}=1.96\right)$, os números de tensiômetros necessários nas três condições de potencial matricial de água são: 20, 14 e 03, respectivamente. Estes dados mostram que, para um mesmo nível de precisão, mais tensiômetros são necessários para medida em solo úmido (potencial menos negativo) do que em solo seco. E exatamente em condições de solo úmido, quando os fluxos de água assumem os maiores valores, é que são necessárias estimativas mais corretas. Tudo indica que $o$ número de 25 tensiômetros, previamente escolhido, foi o ideal, mesmo para o caso de solo mais úmido. Abaixando o nível de exigência, isto $e ́$, para $10 \%$ de desvio, o número de tensiômetros é bem menor, 5,3 e 1 respectivamente.

A Figura 5 mostra os gradientes de potencial total da água, calculados pela equação (1), para as mesmas datas. Vê-se que os gradientes oscilam em torno de $1.5,1.7$ e 1.2 para as condições de solo cada vez mais seco. A totalidade dos gradientes $\varepsilon$ maior que zero (positivos), indicando fluxos descendentes.

Da mesma forma como se procedeu com $h$, foram elaborados autocorrelogramas e semivariogramas, para verificar a existência de uma dependência espacial no gradiente de potencial $\nabla H$ (Figuras 6 e 7). Estas figuras mostram, da mesma forma, não haver dependência espacial.

A Figura 8 mostra os histogramas de freqüência e os gráficos de probabilidade normal, para os dados de gradiente de potencial da Figura 5. Neste caso, apenas os dados referentes à situação intermediária de umidade de solo parecem se aproximar mais da distribuição normal. Da mesma forma como no caso de $h$, foi também feita a análise de variância dos dados de $\nabla \mathrm{H}$, assumindo distribuição normal (TABELA 2). Vê-se claramente que os coeficientes de variação são bem maiores que no caso de $h$, sendo da ordem de $30 \%$ para as três condições de solo. Conseqüentemente o número de medidas $\mathbf{N}$ necessário para obter nova média dentro de limites pré estabelecidos, é bem maior. Na TABELA 2 verifica-se que o $N(5 \%)$ é proibitivo e que até o $\mathrm{N}(10 \%)$ está acima dos 25 pontos utilizados neste trabalho. Daí a conclusão de REICHARDT et al. (1993a e 1993b) de que é praticamente impossível determinar fluxos de água em condições de campo, pelo uso da equação de Darcy.

\section{CONCLUSÕES}

À medida do potencial matricial da água neste solo, feita com tensiômetros, em camadas profundas do solo, tem coeficientes de variação da ordem de 5 a $12 \%$, sendo tanto menor quanto mais seco o solo. $O$ número de pontos de medida do potencial matricial, para obter um valor médio dentro de $10 \%$ do valor esperado é 5 . Nas mesmas condições para medidas de gradiente de potencial total da água, os coeficientes de variação são da ordem de $30 \%$, independentemente da umidade do solo, e o número de pontos de medida está em torno de 50. A variabilidade espacial dos dados de gradiente de potencial total é muito alta, a ponto de compro-meter o uso da equação de Darcy na estimativa de fluxos de água no solo em condições de campo.

\section{REFERÊNCIAS BIBLIOGRÁFICAS}

REICHARDT, K.; LIBARDI, P.L.; MORAES, S.O.; TURATTI, A.; VILLAGRA, M.M. Soil spatial variability and its implications on the establishment of water balances. In: INTERNATIONAL CONGRESS OF SOIL SCIENCE, 14., Kioto, 1990. Proceedings... Amsterdam: ISSS, 1990. p.141-148.

REICHARDT, K.; BACCHI, O.O.S.; VILLAGRA, M.M. Estimativa de fluxos de água em solos não saturados. Bragantia, Campinas, v.52, n.1, p.83-87, 1993a.

REICHARDT, K.; BACCHI, O.O.S.; VILLAGRA, M.M.; TURATTI, A.L.; PEDROSA, Z.O. Hydraulic variability in space and time in a dark red latosol of the tropics. Geoderma, Amsterdam, v.60, p.159-168, $1993 b$.

VILLAGRA, M.M.; MATSUMOTO, O.M.; BACCHI, O.O.S.; MORAES, S.O.; LIBARDI, P.L.; REICHARDT, K. Tensiometria e variabilidade espacial em terra roxa estruturada. Revista Brasileira de Ciencia do Solo, Campinas, v.12, p.205-210, 1988.

WARRICK, A.W.; NIELSEN, D.R. Spatial variability of soil physical properties in the field. In: HILLEL, D. ed. Application of soil physics. New York. Academic Press, 1980. 350p.

Enviado para publicação em 15.07 .93

Aceito para publicação em 01.03.94 